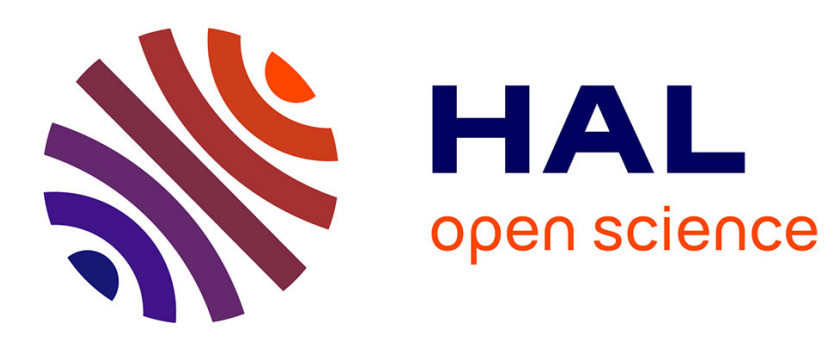

\title{
Design of a soft sensor for the oscillatory failure detection in the flight control system of a civil aircraft
}

Do Hieu Trinh, Benoît Marx, Philippe Goupil, José Ragot

\section{To cite this version:}

Do Hieu Trinh, Benoît Marx, Philippe Goupil, José Ragot. Design of a soft sensor for the oscillatory failure detection in the flight control system of a civil aircraft. International Symposium on Industrial Electronics, ISIE 2010, Jul 2010, Bari, Italy. 10.1109/ISIE.2010.5637753 hal-00498091

\section{HAL Id: hal-00498091 https://hal.science/hal-00498091}

Submitted on 25 Nov 2021

HAL is a multi-disciplinary open access archive for the deposit and dissemination of scientific research documents, whether they are published or not. The documents may come from teaching and research institutions in France or abroad, or from public or private research centers.
L'archive ouverte pluridisciplinaire HAL, est destinée au dépôt et à la diffusion de documents scientifiques de niveau recherche, publiés ou non, émanant des établissements d'enseignement et de recherche français ou étrangers, des laboratoires publics ou privés. 


\title{
Design of a soft sensor for the oscillatory failure detection in the flight control system of a civil aircraft
}

\author{
Do Hieu Trinh*, Benôit Marx*, Philippe Goupil ${ }^{\dagger}$, and José Ragot* \\ ${ }^{*}$ Centre de Recherche en Automatique de Nancy \\ UMR 7039 - Nancy-Université, CNRS, \\ 2 Avenue de la forêt de Haye, 54516 Vandouvre, France \\ Email: \{Do-Hieu.Trinh,Benoît.Marx,Jose.Ragot $\}$ @ensem.inpl-nancy.fr \\ ${ }^{\dagger}$ EDYC-CC Flight Control System, AIRBUS \\ 316 route de Bayonne, 31060 Toulouse Cedex 09, France. \\ Email: Philippe.Goupil@airbus.com
}

\begin{abstract}
The objective of this study is the detection of oscillatory failure (unknown and bounded) that may affect the control system of a control surface (CS) of a civil aircraft. Beside the fact that the failure may result in additional loads on the aircraft structure, it can excite a resonance phenomenon. Early and robust detection of this type of failure is therefore a need for structural design objectives as well as for the controllability of the aircraft. From an analytical model of the actuator and its control loop, a so-called failure-free model translating the behavior of the system in the absence of failure is developed, as well as failure models corresponding to system behaviors in the occurrence of different types of failure. This allows generating residual signals for failure detection.
\end{abstract}

Index Terms-Soft sensor, Fault Detection and Isolation, Oscillatory Failure, Flight Control System

\section{INTRODUCTION}

The safe operation of a physical process can be harmed on the occurrence of faults, these faults may affect the process itself or its conduct bodies. This observation has naturally led to the implementation of surveillance systems whose objective is to be able at any moment, to provide operating status of the various organs constituting the system. When a fault occurs, it must be detected as soon as possible, even where all observed signals remain in their allowable limits. It must then be located and its cause identified. Thus, the steps of observation and monitoring must be assisted by a "smarter" step.

This step, called supervision, uses all available information through an implicit or explicit model. Here, the goal is the detection of oscillatory failures affecting a flight control system (FCS) -specifically an aileron- of an aircraft. For these oscillatory failures, airworthiness regulations applied worldwide by all manufacturers require precaution designed to detect and to accommodate these failures (see [3]). Software embedded on the Airbus A380, for example, is entirely compliant to the current regulations. However, improvements ([1], [2]) could be used for the next generation of aircraft from European manufacturer to accompany the future technological innovations and meet changing regulations. That is the purpose of this study. Examples of oscillatory failures detection in other areas can be found in [4], [5]. Note also that these oscillatory failures are different from Pilot-Induced Oscillations (PIO) caused by the pilot (see [7], [8]). In the following, some elements leading to a methodology to detect such failures are presented, which are based on existing sensors and on soft (or virtual) sensors capable of reconstructing some informations through a model.

The principle of supervision is presented in the section II and the design of a soft sensor for the oscillatory failure case detection in the section III. The section IV tackled the problem of fault detection and isolation (FDI) by the test of standard deviation. This problem is treated in the section $\mathrm{V}$ by the test of correlation. Conclusion and perspectives end the paper.

\section{MODELING OF STUDIED SYSTEM}

The chosen principle is based on testing the adequacy of available measures in a CS servo-loop towards its model. Thus, it is necessary to establish the model of the system, generating through this model and the available measures an indicator of failure. This indicator must be analyzed to detect the presence of this failure as soon as possible. A model called failure-free can be proposed simulating the system behavior in the absence of failure. Failure models corresponding to system behaviors in occurrence of oscillations can also be established. Two kinds of failures must be detected: "liquid failure" and "solid failure" as a disturbing signal is superimposed on or replaces the control signal [1]. The probable sources of oscillatory failure are presented in figure 1. In this application, the characteristic variables of CS servo-loop are given in the table I.

The failure-free model $M_{b}$ is described structurally as follows:

$$
M_{b}=\left\{\begin{array}{l}
\dot{x}_{b}(t)=V_{0}(t) \sqrt{\frac{S \Delta P_{i}(t)+\operatorname{sign}\left(V_{0}(t)\right) F_{a}(t)}{S \Delta P_{r e f}+K_{a}(t) V_{0}^{2}(t)}} \\
V_{0}(t)=K(u(t)-x(t-\tau)) \\
\Delta P(t)=f_{1}\left(x_{d}(t)\right), \quad K_{a}(t)=f_{2}\left(x_{d}(t)\right) \\
F_{a}(t)=f_{3}\left(M_{a}(t), x_{d}(t), V_{a v, x}(t)\right), \\
x_{d}(t)=f_{4}(x(t), \tau)
\end{array}\right.
$$




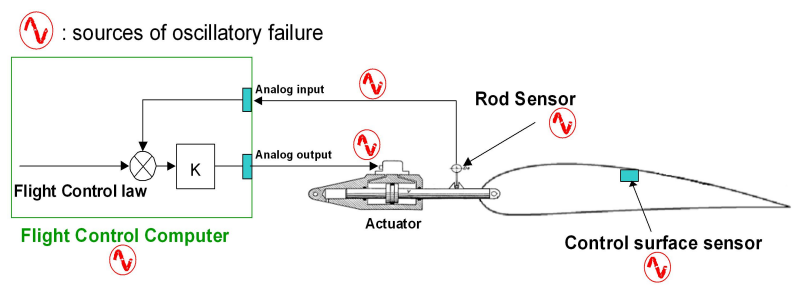

Fig. 1. The probable sources of oscillatory failure

\begin{tabular}{|c|c|}
\hline$x$ & position of the rod of CS actuator (aileron) \\
\hline$u$ & position's order of CS actuator \\
\hline$F_{a}$ & aerodynamic forces applied on the CS \\
\hline$\Delta P$ & $\begin{array}{l}\text { difference of hydraulic pressure in the input of the FCS } \\
\text { actuator }\end{array}$ \\
\hline$K_{a}$ & $\begin{array}{l}\text { damping coefficient of adjacent actuator (in the case of } 2 \\
\text { actuators per CS) }\end{array}$ \\
\hline$\Delta P_{\text {ref }}$ & pressure of reference \\
\hline$\tau$ & transmission delay of the sensor \\
\hline$S$ & surface area of the actuator's piston \\
\hline$K$ & control gain \\
\hline$V_{0}$ & speed computed by flight control computer \\
\hline$x_{d}$ & position (in degrees) of the CS \\
\hline
\end{tabular}

TABLE I

CHARACTERISTIC VARIABLES OF SYSTEM

where the structure of functions $f_{i}($.$) are not detailed here.$ The quantities $\Delta P(t), K_{a}(t)$ and $F_{a}(t)$ play the role of disturbance of which one can know the domain of variation. Failure models of solid and liquid types take the following forms respectively:

$$
\begin{aligned}
& M_{s}=\left\{\begin{array}{l}
\dot{x}_{s}(t)=V_{0, s}(t) \sqrt{\frac{S \Delta P_{i}(t)+\operatorname{sign}\left(V_{0, s}(t)\right) F_{a}(t)}{S \Delta P_{r e f}+K a(t) V_{0, s}^{2}(t)}} \\
V_{0, s}(t)=S_{d e f, s}(t)
\end{array}\right. \\
& M_{\ell}=\left\{\begin{array}{l}
\dot{x}_{\ell}(t)=V_{0, l}(t) \sqrt{\frac{S \Delta P_{i}(t)+\operatorname{sign}\left(V_{0, \ell}(t)\right) F_{a}(t)}{S \Delta P_{r e f}+K_{a}(t) V_{0, \ell}^{2}(t)}} \\
V_{0, \ell}(t)=K\left(u(t)-x_{\ell}(t-\tau)\right)+S_{d e f, \ell}(t)
\end{array}\right.
\end{aligned}
$$

where the magnitudes of $\Delta P(t), K_{a}(t)$ and $F_{a}(t)$ depend on flight scenario; $S_{d e f}(t)$ represents the oscillatory failure signal of unknown frequency, but characterized by a known frequency range.

The principle of supervision, which is therefore to determine at every moment, which mode of the system $M_{b}, M_{s}$ or $M_{\ell}$ is active, is the subject of Section III.

\section{DESIGN OF A SOFT SENSOR FOR THE OSCILLATORY FAILURE DETECTION}

The evolution of outputs noted respectively $x_{b}, x_{s}$ and $x_{\ell}$ are computed by integrating the equations related to the three modes of operation of CS. In this case, one speaks about soft sensor, because the simulation provides information comparable to what gives a physical sensor, under condition that the model is well representative of the system. This allows to propose a diagnostic strategy summarized in the table (II).

The comparison between the outputs of these soft sensors and the growths measured by physical sensors results in three

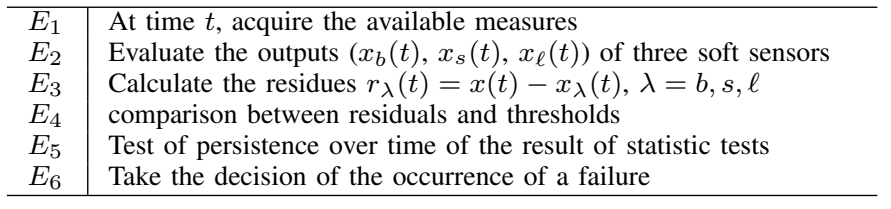

\section{TABLE II}

STRATEGY FOR FAULT DETECTION

residual signals allowing to determine the most representative model of the behavior of the CS and thus to determine the type of the potentially occurring failure. Note that one of the major difficulties in the implementation of this technique is due to the fact that the physical system is subjected to hardly measurable disturbances $\left(\Delta P(t), K_{a}(t)\right.$ and $\left.F_{a}(t)\right)$. In [2], the authors have shown that $\Delta P(t)$ and $F_{a}(t)$ cannot be identified simultaneously and they have chosen to set $\Delta P(t)$ to its most likely value then identify $K_{a}(t)$ and $F_{a}(t)$. Taking into account the complexity of the estimation of $K_{a}(t)$ and $F_{a}(t)$ as well as the limited power of the flight control computer, the model was simplified by fixing the three perturbations $\Delta P(t), K_{a}(t)$ and $F_{a}(t)$ with fixed nominal values ${ }^{1}$. From equation (1), the corresponding evolution of the output $x_{b}(t)$ is:

$$
M_{b}=\left\{\begin{array}{l}
\dot{x}_{b}(t)=V_{0}(t) \sqrt{\frac{S \Delta P_{b}+\operatorname{sign}\left(V_{0}(t)\right) F_{a b}}{S \Delta P_{r e f}+K_{a b} V_{0}^{2}(t)}} \\
V_{0}(t)=K\left(u(t)-x_{b}(t-\tau)\right)
\end{array}\right.
$$

Figure 2 shows the output of the nonlinear model and that of its simplified model; the low amplitude of the difference between the two outputs justifies the use of simplified model.
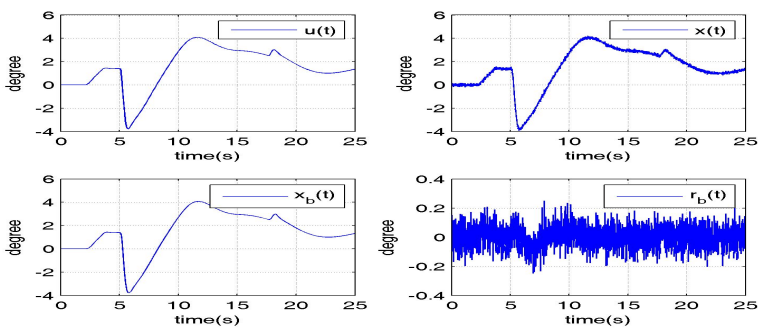

Fig. 2. Validation of simplified model

Constant values are also chosen for the pertubations in the solid and liquid failure models (2 and 3) In following the isolation of an oscillation of 0.5 degree amplitude and 1.5 $\mathrm{Hz}$ frequency will be considered. This case corresponds to an oscillatory failure signal $S_{d e f}(t)$ described by:

$$
\begin{array}{ll}
\text { For the solid failure: } & S_{d e f, s}(t)=0.448 \sin (3 \pi t) \\
\text { For the liquid failure: } & S_{\text {def, },}(t)=1.07 \sin (3 \pi t)
\end{array}
$$

The FD procedure described in table II is applied, using the model of oscillatory failure signal (5) with the failure models (2 and 3) (the sampling period is $0.01 s$ ).

${ }^{1}$ numerical values of the system parameters are not given here for the sake of confidentiality 


\section{FAUlT DETECTION BY STANDARD DEVIATION TEST}

\section{A. Residual generation}

With the actual measurement of the CS position and outputs corresponding to the modes of operation of the system, three residuals are obtained as shown in figure 3.

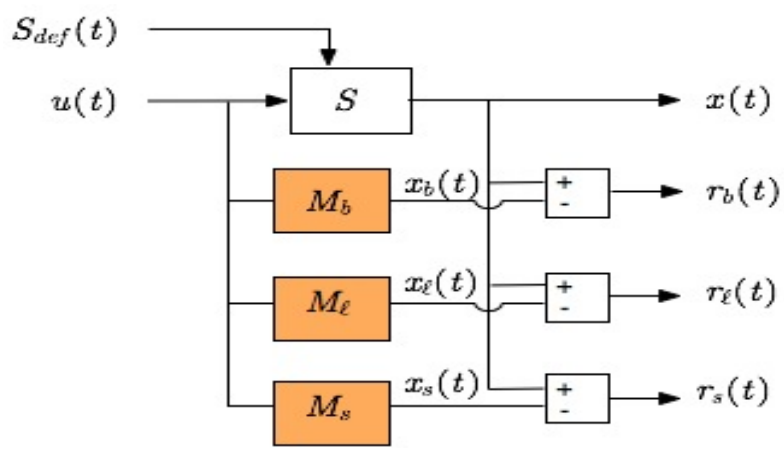

Fig. 3. Bank of residues for the detection of failure

Figure 4, represents the residuals $r_{b}(t), r_{s}(t)$ and $r_{\ell}(t)$ in the case without failure. In the absence of failure, the amplitude of the residual $r_{b}(t)$ is clearly limited to approximately $0.2 \mathrm{deg}$; however, residuals $r_{s}(t)$ and $r_{\ell}(t)$ oscillate with a significantly greater amplitude.
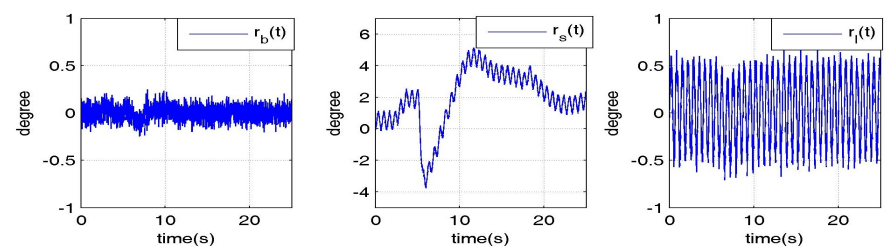

Fig. 4. Residuals $r_{b}(t), r_{s}(t)$ and $r_{\ell}(t)$ : case without failure
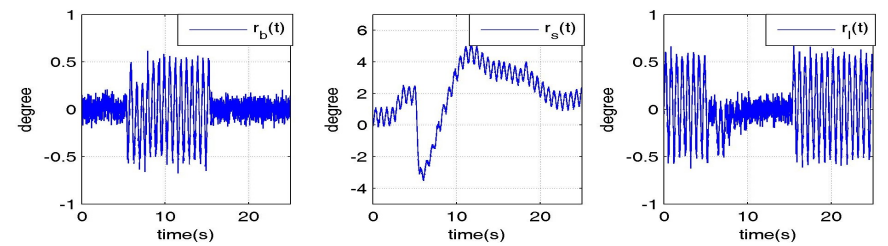

Fig. 5. Residuals $r_{b}(t), r_{s}(t)$ and $r_{\ell}(t)$ : case of liquid failure
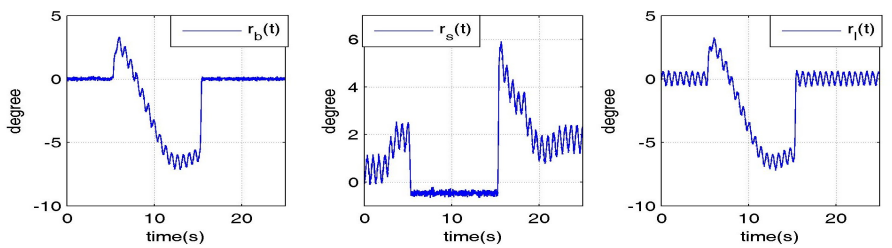

Fig. 6. Residuals $r_{b}(t), r_{s}(t)$ and $r_{\ell}(t)$ : case of solid failure

Figure 5 (resp. figure 6) represents the residuals in the case of liquid failure (resp. solid failure). The failure is simulated between 5.3 and $15.3 \mathrm{~s}$. There is an increase of the variation of the residue $r_{b}(t)$ and a reduction in the variation of the residue $r_{\ell}(t)$ (resp. residue $r_{s}(t)$ ) in the presence of the liquid failure (resp. solid failure). These residues are used for the FDI procedure, the signatures of the residues being well differentiated according to the type of failure.

The previous qualitative visual study showed the ability of three residues to recognize the actual operational situation. In the next section, the quantitative analysis of residues confirms this study and shows how the recognition is processed from a numerical point of view.

\section{B. Generation of failure indication}

The standard deviation measures the dispersion of data set around its mean value, its variations indicate the occurrence or disappearance of a failure. For a residue $r(t)$, this deviation may be calculated over a sliding window of appropriate width $N$ as follows:

$$
\left\{\begin{aligned}
\sigma_{r_{\lambda}}(k) & =\sqrt{\frac{1}{N-1} \sum_{m=k-N+1}^{k}\left(r_{\lambda}(m)-\bar{r}_{\lambda}(k)\right)^{2}} \\
\bar{r}_{\lambda}(k) & =\frac{1}{N} \sum_{m=k-N+1}^{k} r_{\lambda}(m)
\end{aligned}\right.
$$

This assessment is carried out on residuals $\left(r_{\lambda}, \lambda=b, s, \ell\right)$ from failure-free model $M_{b}$ and failure models $M_{s}$ and $M_{\ell}$.

\section{Failure detection by standard deviation test}

Thanks to deviation, the detection of the operational mode and therefore failure may be performed as summarizes the algorithm 1. The principle of this algorithm is to evaluate the relationship between the calculated deviations over sliding windows of appropriate dimensions with the initial deviations (calculated in the absence of failure).

Algorithm 1: Failure detection by standard deviation test

1) Initialization : Calculate the initial deviations $\sigma_{r_{b}, 0}, \sigma_{r_{\ell}, 0}$ and $\sigma_{r_{s}, 0}$.

2) Calculate the deviations $\sigma_{r_{\lambda}}(k)$ over sliding windows

3) Onset of failure: If the failure was not yet detected and that for a period of time $\sigma_{r_{b}}(k) \geq 2 \sigma_{r_{b}, 0}$,

- If $\sigma_{r_{\ell}}(k) \leq 0.5 \sigma_{r_{\ell}, 0}$, then the occurrence of the liquid failure is confirmed.

- If $\sigma_{r_{s}}(k) \leq 0.5 \sigma_{r_{s}, 0}$, then the occurrence of the solide failure is confirmed.

4) Disappearance of the failure: If a failure has already been detected and that for a period of time:

- $\sigma_{r_{b}}(k) \leq 1.5 \sigma_{r_{b}, 0}, \sigma_{r_{\ell}}(k) \geq 0.75 \sigma_{r_{\ell}, 0}$ and $\sigma_{r_{s}}(k) \geq 0.75 \sigma_{r_{s}, 0}$

then the disappearance of the failure is confirmed.

The result of failure detection by the algorithm 1 is illustrated by figures 7, 8 and 9 for the failure free case, the liquid case and the solid case respectively.

The failure flag noted Ind is 1 if the liquid failure is detected, 2 if the solid failure is detected and 0 if no failure is detected. The quantity $r_{S t d}, b$ (resp. $r_{S t d}, s$ and $r_{S t d}, \ell$ ) is the ratio between the calculated deviation on $r_{b}(t)$ (resp. $r_{s}(t)$ and $\left.r_{\ell}(t)\right)$ and the initial standard deviation. 

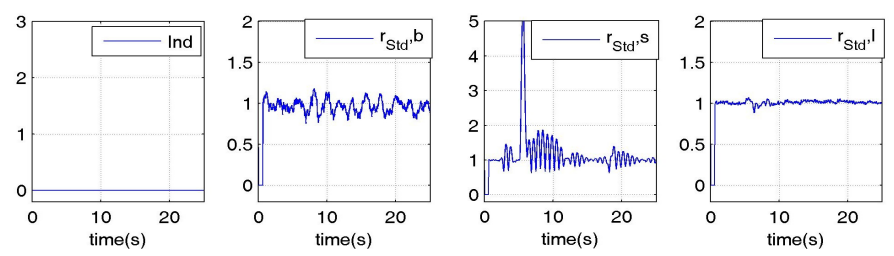

Fig. 7. Result of the detection: case without failure
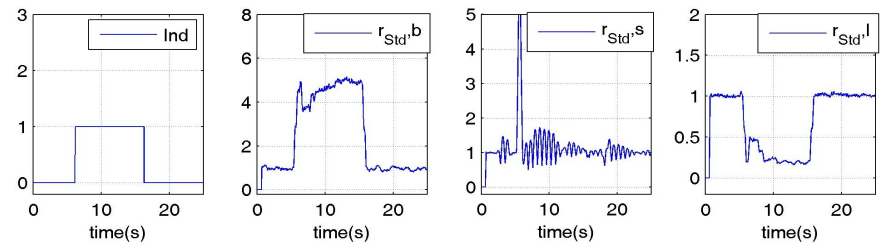

Fig. 8. Result of the detection: case of liquid failure
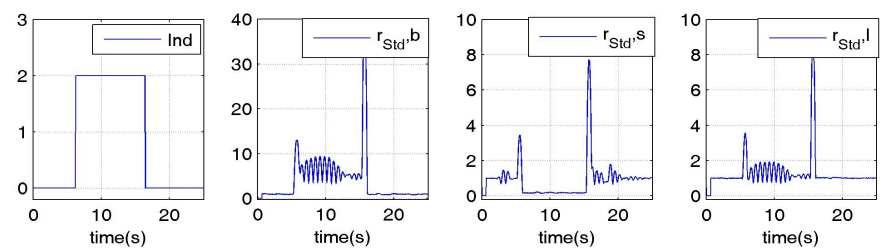

Fig. 9. Result of the detection: case of solid failure

\section{Discussion on failure detection by standard deviation test}

The outcomes of algorithm 1 show that the failure is detected and identified at approximately 1.5 oscillation periods after its occurrence $(0.89$ s for the liquid failure case and 0.93 $\mathrm{s}$ for the solid failure case). This result is compliant with the required specifications.

If one focuses only on the failure detection (without isolation), then only the test of standard deviation of the residual $r_{b}(t)$ is needed, without using failure models. In this case, the failure detection condition should be reduced to $\sigma_{r_{b}}(k) \geq$ $1.75 \sigma_{r_{b}, 0}$ for a period of time (algorithm 1). The different examples show that we can detect any solid and liquid failure on the frequency range $[0.5 \ldots 10.0] \mathrm{Hz}$ even at very low amplitude (0.16 degree).

However, if the goal is to detect and isolate all failures that may appear in the control loop, the number of failure models described by equations (2) and (3) must be increased and therefore different frequencies of oscillations must be taken into consideration. Each failure model whose parameters are fixed is specific to a particular solid or liquid failure (of type (5)). With the principle used by the algorithm 1, any solid and liquid failure characterized by a frequency in $[0.5 \ldots 10.0] \mathrm{Hz}$ and an amplitude in [0.5 ..1.0] deg can be detected and isolated for many flight scenarios.

To reduce the number of failure models, another approach is to use a correlation test that is developed in the next section.

\section{FAULT DETECTION BY CORRELATION TEST}

The dysfunction models (2 and 3) allow to study the behavior of the system in the presence of a failure. In the simulation of dysfunction models, by forcing the command to zero, the impact of the failure on the output can be directly identified and estimated. In this way, patterns of failures can be generated offline to be compared to the residue $r_{b}(t)$ or output $x(t)$ to detect and isolate the failure. Figure 10 shows the procedure to be implemented. The first residue $r_{b}(t)$ has already been defined. Signals $f_{i}(t)$ correspond to failures characterized by some specific frequencies (5) whose effect is assessed based on the failure models $\left(M_{s}\right.$ or $\left.M_{\ell}\right)$ thus generating signatures $x_{L i}(t)$ or $x_{S i}(t)$ specific to each of these frequencies. Such frequencies are called "selected" because this work aims at detecting and isolating the failures of these frequencies. These signatures are then compared (by correlation over sliding windows) to the previously evaluated residue $r_{b}(t)$ or output $x(t)$. This principle applies to liquid and solid failure, model $S_{M F}$ is then $M_{\ell}$ or $M_{s}$.

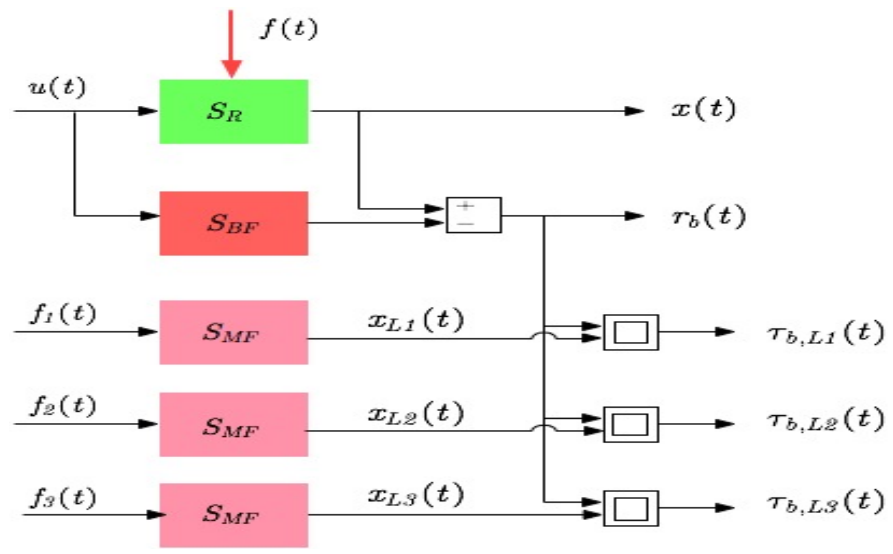

Fig. 10. Generation of residues for correlation test

\section{A. Pattern generation}

In this subsection patterns at $0.5 \mathrm{~Hz}, 1.5 \mathrm{~Hz}$ and $7.0 \mathrm{~Hz}$ are generated from models $M_{s}$ and $M_{\ell}$ by putting the command to zero. As the correlation test does not distinguish the amplitudes of sinusoidal signals, these patterns are generated so that they correspond with the oscillation of $0.75 \mathrm{deg}$. Each pattern is a sequence of length equal to two periods of the failure of the same frequency. The objective is to detect and to isolate these three failures.

1) Patterns of liquid failures: For the liquid failures, the three following patterns are generated (table III)

TABLE III

LIQUID FAILURES

\begin{tabular}{l|l|l}
\hline Pattern & Sequence & Frequency \\
\hline$x_{L 1}$ & 400 samples & $0.5 \mathrm{~Hz}$. \\
$x_{L 2}$ & 135 samples & $1.5 \mathrm{~Hz}$. \\
$x_{L 3}$ & 28 samples & $7.0 \mathrm{~Hz}$. \\
\hline
\end{tabular}

These three patterns $x_{L 1}, x_{L 2}$ and $x_{L 3}$ are presented in figure 11 . They are analyzed by correlation test with the signal $r_{b}(t)$ defined previously by $r_{b}(t)=x(t)-x_{b}(t)$.

The patterns $x_{L 1}, x_{L 2}$ and $x_{L 3}$ are the direct impacts of 

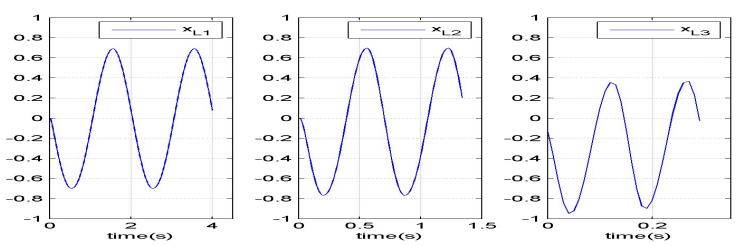

Fig. 11. Patterns $x_{L 1}, x_{L 2}$ and $x_{L 3}$

liquid failures (without the influence of the command) on the output of the system and they are comparable in some way with the residue $r_{b}(t)$ under the presence of a failure. Indeed, the difference $x(t)-x_{b}(t)$ reflects the impact of the failure on the output since the effect of the command on $x(t)$ and $x_{b}(t)$ is canceled by difference.

2) Patterns of solid failures: For the solide failures, the three following patterns are generated (table IV).

TABLE IV

SOLID FAILURES

\begin{tabular}{l|l|l}
\hline Pattern & Sequence & Frequency \\
\hline$x_{S 1}$ & 400 samples & $0.5 \mathrm{~Hz}$. \\
$x_{S 2}$ & 135 samples & $1.5 \mathrm{~Hz}$. \\
$x_{S 3}$ & 28 samples & $7.0 \mathrm{~Hz}$. \\
\hline
\end{tabular}

These three patterns $x_{S 1}, x_{S 2}$ and $x_{S 3}$ are presented in figure 12 . They are analyzed by correlation test with the actual output $x(t)$.
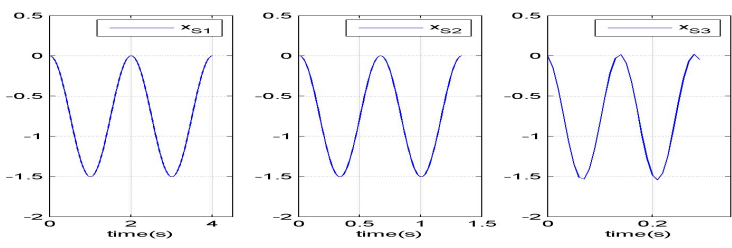

Fig. 12. Patterns $x_{S 1}, x_{S 2}$ and $x_{S 3}$

\section{B. Failure indicator generation}

The correlation between two or more variables is the intensity of the relation that may exist between these variables. A measure of this correlation is obtained by the calculation of the linear correlation coefficient. The linear correlation coefficient between two variables $x$ and $y$ is noted by $r_{x, y}$.

\section{Fault detection by correlation test}

With the correlation test, FD can be performed as summarized in Algorithm 2. The principle of this algorithm is to compute the linear correlation coefficients over a sliding window, on the one hand between the residue $r_{b}(t)$ with signals $x_{L 1}, x_{L 2}$ and $x_{L 3}$ which represent liquid failures; on the other hand between the output $x(t)$ with signals $x_{S 1}, x_{S 2}$ and $x_{S 3}$ which represent solid failures. If one of these coefficients calculated over a sliding window exceeds a threshold a certain number of times within a limited time, a failure is detected.
Algorithm 2: Fault detection by correlation test

1) Initialization :

- Read the patterns $x_{L 1}, x_{L 2}, x_{L 3}, x_{S 1}, x_{S 2}$ and $x_{S 3}$ from a file previously created.

- Define a table $P=\left[\begin{array}{lll}400 & 135 & 28\end{array}\right]$ and one threshold $V_{s}=0.6$.

2) Perform calculations of linear correlation coefficients for each $k^{t h}$ sampling step:

- Calculate $r_{r_{b(k-P(i)+1: k)}, x_{L i}}$ for $i=1,2,3$

- Calculate $r_{x_{(k-P(i)+1: k)}, x_{S i}}$ for $i=1,2,3$.

3) Evaluate the linear correlation coefficients by counting exceedances:

- If a coefficient is greater than $V_{s}$ or smaller than $-V_{s}$, the number of so-called overruns associated with this coefficient is increased by one unit.

- If no exceedance were observed within a limited time, the number of overruns is set to zero.

4) Onset of failure: If the failure has not yet been detected and if one of the overruns is greater than or equal to 4; then:

- the onset of failure is confirmed.

- The nature of the failure (liquid or solid) as well as its frequency are indicated by the pattern $x_{L i}$ or $x_{S i}$ whose number of exceedances was observed with its correlation coefficient. If it is a pattern $x_{L i}$, the failure is liquid; if it is a pattern $x_{S i}$, the failure is solid. The value of $i$ indicates the frequency of the failure.

5) Disappearance of the failure: If a failure has already been detected and no exceedance was observed within a limited time; then

- the failure is declared to have disappeared.

The correlation coefficients calculated during the time are shown first in figure 13 for the case without failure. The result of failure detection by the algorithm 2 is shown in figures 14 , 15 and 16. Figure 14 (resp. 15 and 16) represents the result obtained with the liquid failure of $0.5 \mathrm{~Hz}$ frequency (resp. the liquid failure of $1.5 \mathrm{~Hz}$ frequency and with the solid failure of $7.0 \mathrm{~Hz}$ frequency). Failures are simulated between 5.3 and 15.3 seconds.
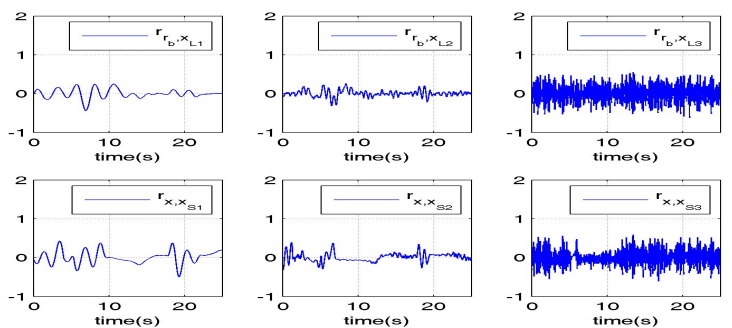

Fig. 13. Calculated correlation coefficients : case without failure

The first column represents the correlation coefficient which led to the failure detection $\left(r_{r_{b}, x_{L 1}}, r_{r_{b}, x_{L 2}}\right.$ and $r_{x, x_{S 3}}$ respectively). The indicator of nature of the failure is noted by Nat in the second column. If $\mathrm{Nat}=1$, a liquid failure is detected, 

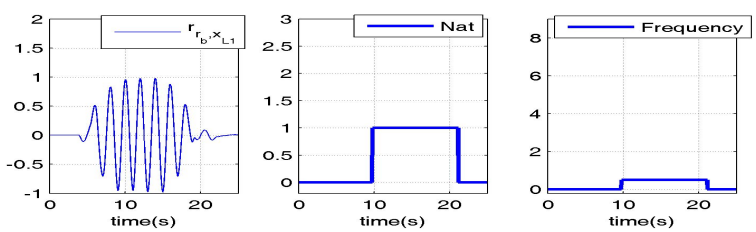

Fig. 14. Result of detection : liquid failure of $0.5 \mathrm{~Hz}$ frequency
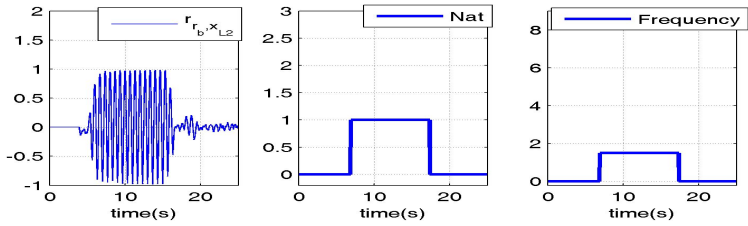

Fig. 15. Result of detection : liquid failure of $1.5 \mathrm{~Hz}$ frequency
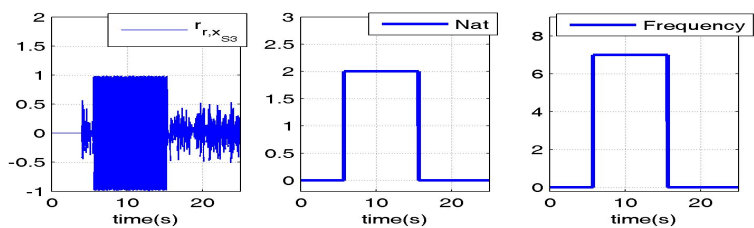

Fig. 16. Result of detection : solid failure of $7.0 \mathrm{~Hz}$ frequency

if Nat $=2$, a solid failure is detected. The frequency of the failure is indicated in the third column. With this method, the detection and isolation of failure can be performed in less than three periods of the failure, which is in fact compliant with the required specifications.

\section{Discussion on the failure detection by correlation test}

Failure detection by correlation test has reduced significantly the number of failure models compared to deviation test. In fact, boarding only failure-free model in flight control computer to generate the residue $r_{b}(t)$ is sufficient. All the pattern of liquid and solid failures are generated in advance and stored. Different treated flight scenarios show that any solid and liquid failure characterized by frequencies in the frequency range $[0.5 \ldots 10.0] H z$, even at low amplitude $(0.16 \mathrm{deg})$ can be detected and isolated. However, this analysis does not estimate the amplitude of the failure.

It should be noted that, in its current version, the algorithm 2 uses 6 correlation tests at every step of simulation (2 types of default, 3 selected frequencies). Although the calculations are simple and need basic operators, it is possible to substantially reduce the computational load :

- The patterns $x_{L 1}, x_{L 2}, x_{L 3}, x_{S 1}, x_{S 2}$ and $x_{S 3}$ are determined by the type of failure. Their means and standard deviations over a window can be calculated offline and stored.

- Means, standard deviations of the output $x(t)$ and the residue $r_{b}(t)$ calculated over a window can be performed recursively when moving a step time of the observation window.

- The covariance between a reference pattern and a signal $x(t)$ or $r_{b}(t)$ over a window can also be calculated in recursive way.
These recurrences are easy to establish, so the correlation tests can be carried out with a reasonable computational load.

\section{CONCLUSION}

This paper addresses the problem of detecting the oscillatory failure in the control system of a control surface of a civil aircraft. Two fault detection methods are proposed, based on a simplified model validated regarding the nonlinear models usually used. Any solid and liquid failure in the frequency range $[0.5 \ldots 10.0] \mathrm{Hz}$ can be detected by standard deviation test, as well as any solid and liquid failure of selected frequencies by correlation test. Both methods have been successfully tested for a variety of flight scenarios, even with failures of low amplitude (0.16 degree). Future works will consist in extending these methods to other control surfaces (rudder or elevator), by trying to reduce the complexity as well as the number of failure models. The improvement of the robustness of the correlation test for the failures of frequencies neighboring the selected ones is also to be considered.

Acknowledgement. The authors would like to thank the French Research Foundation for Aviation and Space, for its support to the SIRASAS project.

https://extranet.ims-bordeaux.fr/External/SIRASAS/

\section{REFERENCES}

[1] L. Lavigne, A. Zolghadri, P. Goupil, and P. Simon, Robust and early detection of oscillatory failure case for new generation Airbus aircraft, in AIAA-2008-7139, AIAA Guidance, Navigation and Control Conference, 2008.

[2] L. Lavigne, A. Zolghadri, P. Goupil, and P. Simon, Oscillatory failure case detection for new generation Airbus aircraft : a model-based challenge, in 47th IEEE CDC Conf, Cancun, Mexico, Dec. 2008.

[3] P. Goupil. Oscillatory Failure Case detection in A380 Electrical Flight Control System by analytical redundancy. To appear in Control Engineering Practice, 2009.

[4] P.K. Dash, R.K. Jena, G. Panda, A. Routray. An extended comples Kalman filter for frequency measurement of distorded signals, IEEE Trans. on Instrumentation and Measurement, vol.49 (4), 746-753, 2000.

[5] Q.P. He, J. Wang, M. Pottmann, S.J. Qin. A Curve fitting method for detecting valve striction in oscillating control loops, Industrial \& Engineering Chemistry Research, 46 (13), 4549-4560, 2007.

[6] J.S. Yee, Jian L. Wang, B. Jiang. Actuator Fault Estimation Scheme for Flight Applications. Actuator Fault Estimation Scheme for Flight Applications, 124 (4), 701-704, 2002.

[7] G. Jeram, J. Prasad. Fuzzy logic detector for aircraft pilot coupling and pilot induced oscillation (PIO). In Proceedings of the 59th American Helicopter Society Annual Forum, Phoenix, AZ, May 6-8, 2003.

[8] D. G. Mitchell, D. H. Klyde. Recommended Practices for Exposing Pilot-Induced Oscillations or Tendencies in the Development Process. In USAF Developmental Test and Evaluation Summit, Woodland Hills, California, 16 - 18 November 2004. 